\title{
Engineering $T$ cells for customized therapeutic responses
}

Chimeric antigen receptor (CAR)

$\mathrm{T}$ cells are a promising type of cancer immunotherapy in which T cells are engineered to express receptors for tumour-specific antigens, thereby unleashing a cytotoxic immune response against cancer cells.

However, the effect of such treatment is limited to the natural responses of $\mathrm{T}$ cells downstream of $\mathrm{T}$ cell receptor activation, and toxicity remains a key concern. In a recent study, Roybal et al. report the use of synthetic Notch (synNotch) receptors to engineer $\mathrm{T}$ cells with customized therapeutic response programmes that could have greater efficacy and safety than CAR T cells and might extend the utility of $\mathrm{T}$ cell-based therapies to diseases beyond cancer.

The authors used their previously developed platform to generate synNotch receptors. Like the native

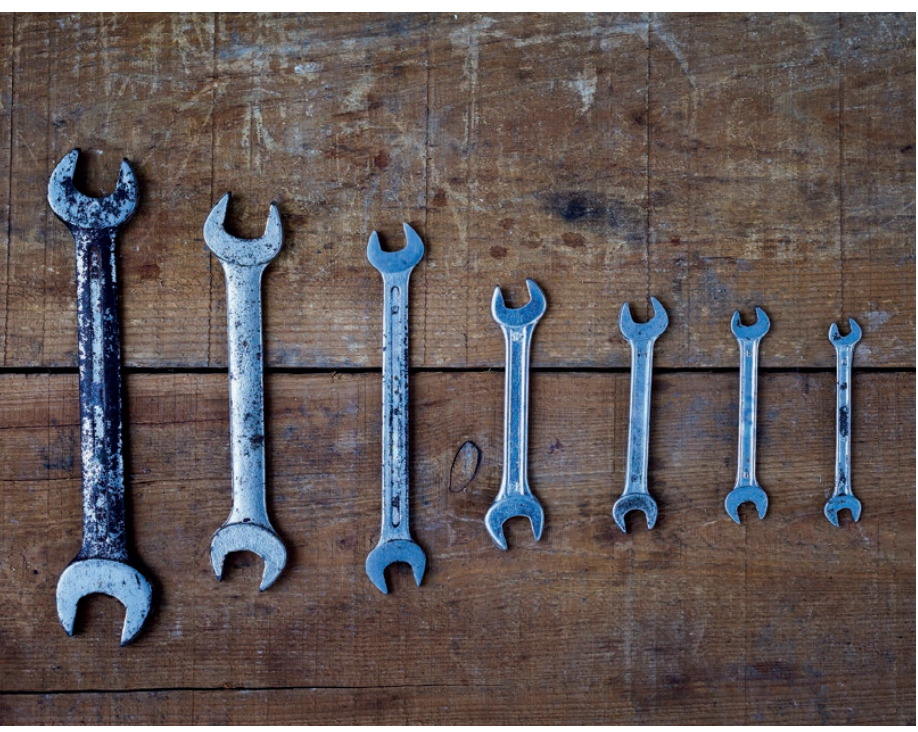

EyeEm/Alamy Stock Photo version, synNotch receptors have an extracellular antigen-binding domain, a core regulatory region and an intracellular domain that is released from the receptor complex upon antigen binding and regulates gene transcription. Crucially, the extracellular and intracellular domains of synNotch receptors are custom-made to enable receptor binding to an antigen of choice and subsequent activation of selected genes.

In vitro, synNotch-engineered $\mathrm{T}$ cells were capable of driving a range of therapeutically useful responses following co-culture with cells expressing the cognate antigen for the extracellular domain of the synNotch receptor. These included antitumour responses, such as induction of 'à la carte' cytokine profiles or skewing the differentiation of the $\mathrm{T}$ cells to an antitumour T helper 1 $\left(\mathrm{T}_{\mathrm{H}} 1\right)$ fate, as well as production of the custom therapeutic TRAIL (TNF-related apoptosis-inducing ligand), therapeutic antibodies, bispecific $\mathrm{T}$ cell engagers or adjuvants.

In addition, the authors generated synNotch-expressing $\mathrm{T}$ cells that produced the immunosuppressive agents interleukin-10 (IL-10) and programmed cell death 1 ligand 1 (PDL1), which could be useful for treating autoimmune diseases.

For proof-of-concept studies in vivo, Roybal et al. used a xenograft mouse model in which a CD19- tumour and a CD19+ tumour were implanted in the left and right flanks, respectively. 4 days later, the researchers intravenously injected the mice with $\mathrm{T}$ cells engineered with CD19-specific synNotch receptors containing response elements in control of IL-2 expression. Tumours were harvested after 6 days, and only T cells in CD19 ${ }^{+}$target tumours were found to express IL-2, although the authors note that the ability of synNotch T cells to infiltrate the tumours could be improved.

Last, the researchers tested synNotch $\mathrm{T}$ cells engineered to bind green fluorescent protein (GFP) and thereupon produce the bispecific therapeutic antibody blinatumomab. This antibody binds to CD19 and the CD3 chain of the T cell receptor, thereby helping to bring tumour cells and $\mathrm{T}$ cells into closer proximity. Tumours expressing both the GFP 'priming antigen' and the CD19 'killing antigen' were selectively cleared 25 days after intravenous injection with the synNotch T cells. By contrast, bilateral tumours expressing only the blinatumomab antigen (CD19) continued to grow. These findings indicate the utility of synNotch T cells for spatially localized production of therapeutics at solid tumours.

Importantly, the versatility of the synNotch platform means that the diverse $\mathrm{T}$ cell responses described in this paper are likely to represent only a fraction of the technology's full potential.

Katie Kingwell

ORIGINAL ARTICLE Roybal, K. T. et al. Engineering T cells with customized therapeutic response programs using synthetic Notch receptors. Cell 167, 419-432 (2016) 